\title{
Combining Surface Impedance Boundary Conditions with Volume Discretisation in Time-Domain Finite-Element Modeling
}

\author{
J. Gyselinck ${ }^{1}$, P. Dular ${ }^{2,3}$, C. Geuzaine ${ }^{2}$ and R. V. Sabariego ${ }^{2}$ \\ ${ }^{1}$ BEAMS Department, Université Libre de Bruxelles (ULB), Belgium, email: johan.gyselinck@ulb.ac.be \\ ${ }^{2}$ ACE, Dept. of Electrical Engineering and Computer Science, ${ }^{3}$ F.R.S.-.FNRS, University of Liège, Belgium
}

\begin{abstract}
In this paper a hybrid approach for considering massive conducting regions in time-domain finite-element modeling is presented. Surface impedance boundary conditions, developed in the time domain as previously proposed by the authors, are adopted so as to accomodate for high-frequency flux components (in a certain frequency band) without the need for very fine discretisation of the conducting region near its surface. These surface conditions are combined with a coarse FE mesh inside the region for allowing for slowly varying flux components. The correct impedance behaviour can thus be obtained in a wide frequency range, from DC flux on. The approach is briefly demonstrated by means of a simple 1D test case.
\end{abstract}

\section{INTRODUCTION}

Surface-impedance boundary conditions (SIBCs) are widely applied in frequency-domain eddy-current problems for considering massive conducting regions. Several time-domain approaches have been proposed to date [1][2]. The approach in [2] is based on the discretisation of the 1-D half-plane eddy-current problem by means of dedicated basis functions. The latter are pair-wise derived from the analytical frequencydomain solution considering a number of discrete frequencies covering the relevant frequency interval.

\section{HYBRID APPROACH}

The hybrid approach will hereafter be explained by means of a basic 1D geometry: a non-magnetic conducting region $\Omega$ of depth $L=100 \mathrm{~mm}(0 \leq x \leq L)$, with conductivity $\sigma=6 \cdot 10^{7} \mathrm{~S} / \mathrm{m}$, which is either coarsely meshed (20 elements of each $5 \mathrm{~mm}$ length) or finely meshed (reference FE solution).

Considering frequency-domain calculations with the magnetic vector potential $a$, the flux density $b=\operatorname{curl} a$ and the electric field $e=-\partial_{t} a$ are linked together at the surface $x=0$ :

$$
\partial_{x} \boldsymbol{a}=-\frac{1+\boldsymbol{i}}{\delta} \cdot \boldsymbol{a}
$$

with $i=\sqrt{-1}$ and $\delta$ the penetration depth, supposing that $\Omega$ is infinitely deep ( $\delta \ll L$, hypothesis of the SIBC approach).

For comparing globally the different approaches, we will consider the complex value $\boldsymbol{Z}$ that is equal to $1 /(1+\boldsymbol{i})$ if no discretisation error is made and supposing $\delta \ll L$. More precisely, for sake of brevity, we will show $Z_{n}=\sqrt{2} \cdot \operatorname{abs}(\boldsymbol{Z})$, referred to as normalised impedance, and ignore the phase difference (or error).

Let us first look at the SIBCs taking e.g. $f_{1}=100, f_{2}=$ 1000 and $f_{3}=10000$ as discrete frequencies (in Hz). Fig. 1 shows a very good accuracy in the interval $\left[f_{1}, f_{3}\right]$, i.e. $Z_{n}$ close to 1 , with some overshoot on both sides.

This work has been supported by the Belgian Science Policy (IAP P6/21).
For the characteristic $Z(f)$ to drop quicker to zero on the left side of the interval $\left[f_{1}, f_{3}\right]$, a fictitious frequencydependent conductivity $\sigma^{*}(f)$ can be adopted:

$$
\sigma^{*}=\left(1+c_{1} / f^{2}+\ldots\right) \sigma,
$$

with the coefficients $c_{1}, c_{2}, \ldots$ properly choosen.

An example of such a cut-off correction is shown in Fig. 1. Note that the conductivity $\sigma^{*}(f)$ can be taken into account straightforwardly in the time domain (see extended paper).

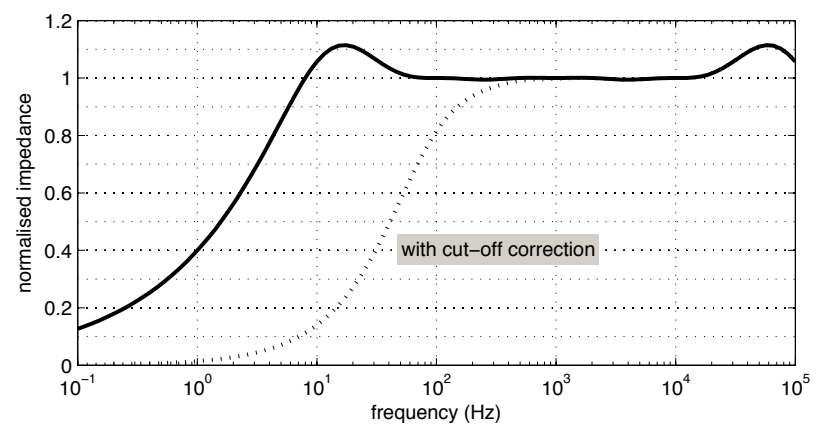

Fig. 1. Normalised impedance versus frequency obtained with SIBC approach (with and without cut-off correction)

Fig. 2 shows the result of combining the SIBC approach and the coarse mesh. Between 0 and $10000 \mathrm{~Hz}$ a good agreement with the reference FE solution is obtained.

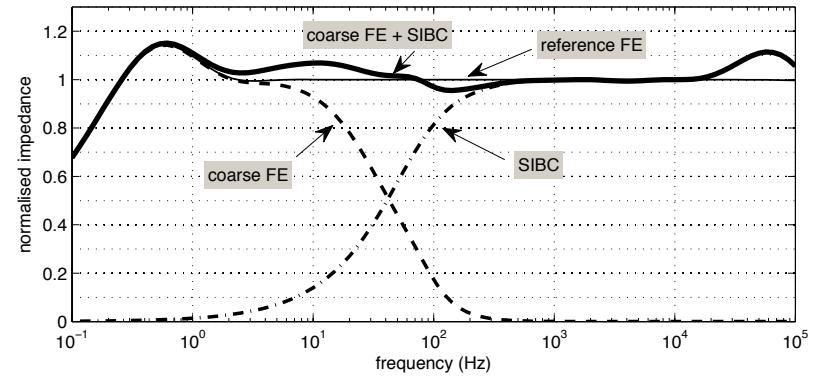

Fig. 2. Normalised impedance versus frequency obtained with fine FE mesh, coarse FE mesh, SIBC approach and hybrid approach approach (with and without cut-off correction)

In the extended paper the practical time-domain implementation of this hybrid approach will be discussed in detail. A 2D application example will be treated.

\section{REFERENCES}

[1] L. Krähenbühl, O. Fabrègue, S. Wanser, M. De Sousa Dias and A. Nicolas, "Surface impedances, BIEM and FEM coupled with 1D non linear solutions to solve 3D high frequency eddy current problems," IEEE Trans. Magn., vol. 33, pp. 1167-1172, March 1997.

[2] J. Gyselinck, P. Dular, C. Geuzaine, R. V. Sabariego, "Surface-Impedance Boundary Conditions in Time-Domain Finite-Element Calculations using the Magnetic-Vector-Potential Formulation," IEEE Trans. Magn., vol. 45, pp. 1280-1283, March 2009. 